\title{
Reflux-related Extraesophageal Symptoms Until Proven Otherwise: A Direct Measurement of Abnormal Proximal Exposure Based on Hypopharyngeal Multichannel Intraluminal Impedance as a Reliable Indicator for Successful Treatment Outcomes
}

\begin{abstract}
Takeshi Suzuki, ${ }^{1}$ Yosuke Seki, ${ }^{2}$ Tomoaki Matsumura, ${ }^{3}$ Makoto Arai, ${ }^{4}$ Toyoyuki Hanazawa, ${ }^{1}$ Yoshitaka Okamoto, ${ }^{5}$ Haruhiko Suzuki, ${ }^{6}$ Kazunori Kasama, ${ }^{2}$ Akiko Umezawa, ${ }^{2}$ Yoshimoti Kurokawa, ${ }^{2}$ and Toshitaka Hoppo ${ }^{7 *}$

${ }^{1}$ Department of Otolaryngology, Head and Neck Surgery, Graduate School of Medicine, Chiba University, Chiba, Chiba, Japan; ${ }^{2}$ Minimally Invasive Surgery Center, Yotsuya Medical Cube, Tokyo, Japan; ${ }^{3}$ Department of Gastroenterology, Graduate School of Medicine, Chiba University, Chiba, Chiba, Japan; ${ }^{4}$ Department of Medical Oncology, Graduate School of Medicine, Chiba University, Chiba, Chiba, Japan; ${ }^{5}$ Chiba Rosai Hospital, Ichihara, Chiba, Japan; ${ }^{6}$ Suzuki ENT Clinic, Narashino, Chiba, Japan; and ${ }^{7}$ Esophageal Institute, Allegheny Health Network, Pittsburgh, $P A, U S A$
\end{abstract}

\section{Background/Aims}

The Lyon Consensus defined parameters based on upper endoscopy and 24-hour combined multichannel intraluminal impedance$\mathrm{pH}(\mathrm{MII}-\mathrm{pH})$, that conclusively establish the presence of gastroesophageal reflux disease (GERD). However, the true role of upper endoscopy and MII-pH to evaluate patients with extraesophageal symptoms (EES) has not been well established. Hypopharyngeal MII (HMII), which directly measures laryngopharyngeal reflux (LPR) events, has been utilized to evaluate patients with EES suggestive of LPR.

\section{Methods}

This was a retrospective study involving patients with EES for $>12$ weeks despite proton pump inhibitor therapy, and had no endoscopic confirmatory evidence for GERD and negative MII-pH. All patients were subsequently referred for further evaluation of EES with "unknown" etiology and underwent laryngoscopy and HMII. Based on HMII, abnormal proximal exposure (APE) was defined as $L P R \geq 1 /$ day and/or full column reflux (reflux $2 \mathrm{~cm}$ distal to the upper esophageal sphincter) $>4$ /day. Patients with APE were offered antireflux surgery (ARS) and the outcome of ARS was objectively assessed using Reflux Symptom Index.

\section{Results}

Of 21 patients with EES which was thought to be GERD-unrelated based on endoscopy and MII-pH, 17 patients (81\%) had APE. Eight patients with APE who had undergone ARS had significant symptomatic improvement in the Reflux Symptom Index score (19.6 \pm 4.9 pre-ARS to $5.8 \pm 1.4$ post-ARS, $P=0.008$ ).

\section{Conclusions}

A conventional diagnostic approach using endoscopy and MII-pH may not be sufficient to evaluate patients with EES suggestive of LPR. HMII is essential to evaluate patients with EES, and APE could be a reliable indicator for successful treatment outcomes.

(J Neurogastroenterol Motil 2022;28:69-77)

Key Words

Hypopharynx; Laryngopharyngeal reflux; Treatment outcome 
Received: October 8, 2020 Revised: February 9, 2021 Accepted: March 8, 2021

(c) This is an Open Access article distributed under the terms of the Creative Commons Attribution Non-Commercial License (http://creativecommons. org/licenses/by-nc/4.0) which permits unrestricted non-commercial use, distribution, and reproduction in any medium, provided the original work is properly cited.

*Correspondence: Toshitaka Hoppo, MD, PhD Esophageal Institute, Allegheny Health Network, 4815 Liberty Avenue, Mellon Pavilion, Suite 439, Pittsburgh PA 15224, USA Tel: +1-724-260-7300,Fax: +1-724-260-7310,E-mail: toshitaka.hoppo@ahn.org

Takeshi Suzuki and Yosuke Seki equally contributed to this work.

\section{Introduction}

Gastroesophageal reflux disease (GERD) is defined as a condition that develops when the reflux of gastric contents into the esophagus causes troublesome symptoms and/or complications, in the Montreal Consensus. ${ }^{1}$ GERD can cause esophageal (typical) and extraesophageal (atypical) symptoms (EES). Typical symptoms include heartburn and regurgitation, while EES are nonspecific, and includes various laryngeal symptoms such as chronic cough and hoarseness, as well as pulmonary and otolaryngological diseases such as laryngitis, asthma, dental erosion, pharyngitis, sinusitis, idiopathic pulmonary fibrosis, and recurrent otitis media. ${ }^{1}$ In particular, laryngopharyngeal reflux (LPR) disease (LPRD) has been considered to be present if the patients have EES, and LPR symptoms have been thought to be caused by direct exposure of gastric contents to the laryngopharynx. Although upper endoscopy is the first examination for GERD to assess the presence of esophageal mucosal injury such as esophagitis and Barrett's esophagus, absence of esophageal mucosal injury cannot exclude GERD. More than half of the patients with GERD have non-erosive reflux disease (NERD). Therefore, it is crucial to objectively evaluate abnormal acid exposure to the distal esophagus, especially in the patients with NERD, and 24-hour esophageal multichannel intraluminal impedance-pH (MII-pH) monitoring is currently considered as the gold standard testing modality. Recently, gastroenterology experts have proposed the Lyon Consensus, which defined parameters on esophageal testing such as upper endoscopy and MII-pH, that can conclusively establish the presence of pathological GERD. ${ }^{3}$ However, a diagnosis of LPRD is still challenging. ${ }^{1}$ Laryngoscopic findings such as hypopharyngeal inflammation are nonspecific, ${ }^{4,5}$ and may be often seen in healthy population without any LPR symptoms. ${ }^{6}$ Although MII-pH is also indicated for the evaluation of EES, a true role of upper endoscopy and MII-pH to evaluate EES was not well established in the Lyon Consensus.

Hypopharyngeal MII (HMII) uses a special impedance catheter to directly measure LPR, and has been utilized to evaluate patients with EES and pulmonary diseases. ${ }^{8,9}$ Based on the normative date established, abnormal proximal exposure (APE) was defined as LPR $\geq 1$ /day and/or full column reflux (FCR) (reflux $2 \mathrm{~cm}$ distal to the upper esophageal sphincter [UES] $)>4 /$ day. ${ }^{10}$ Although the efficacy of HMII to identify patients with LPR symptoms, who would benefit from antireflux surgery (ARS) has been shown, ${ }^{11}$ the use of HMII has been still limited. The objectives of this study are to investigate the benefit of HMII for the evaluation of the patients with persistent EES which was thought to be GERD-unrelated based on endoscopy and MII-pH, and to assess the outcome of ARS in patients with APE as measured by HMII.

\section{Materials and Methods}

\section{Study Design}

This is a retrospective study which was conducted in accordance with the Declaration of Helsinki under the approval of the institutional review board (No. 2356). The patient population in this study included those who presented with predominant or isolated EES (LPR symptoms) that lasted for longer than 12 weeks despite proton pump inhibitor (PPI) therapy, were considered to have EES with GERD-unrelated "unknown" etiology based on no confirmatory evidence for GERD such as Los Angeles (LA) grade $\mathrm{C} / \mathrm{D}$ esophagitis or Barrett's esophagus on the prior upper endoscopy, and negative MII-pH, and were referred for further evaluation of LPR from July 1, 2015 to April 30, 2018. All patients subsequently underwent laryngoscopy and HMII by a single experienced laryngologist (T.S.). EES were defined as globus sensation, chronic cough, dysphonia, abnormal sensation in the mouth (halitosis, dry mouth, and bitter or acidic taste), and of the laryngopharynx. Patients with APE as measured by HMII were offered ARS, and subsequently underwent ARS by a single experienced surgeon (Y.S.). Detailed demographic data, subjective data (Reflux Symptom Index [RSI]), and objective data (endoscopic, MII-pH, and HMII findings) were obtained from the medical records. The outcome of ARS was objectively assessed using RSI before and after ARS. The following examination was similar to that in a recent our study; ${ }^{5}$ as such, the description of the methodology is abbreviated 
below.

\section{Endoscopic Evaluation}

Upper endoscopy was performed by an experienced gastroenterologist. The severity of esophagitis was graded using the LA classification. $^{12}$

Laryngoscopy was performed by an experienced otolaryngologist to evaluate the laryngohypopharyngeal area and to rule out any known etiology of laryngopharyngeal symptoms.

\section{Combined Multichannel Intraluminal Impedance-pH Testing}

MII-pH used the catheter (ZAN-BG-44; Sandhill Scientific Inc, Highlands Ranch, CO, USA) (Fig. 1A), which was positioned $5 \mathrm{~cm}$ above the manometrically defined upper margin of the lower esophageal sphincter.

HMII was performed using the specialized catheter (CAZIBL-55; Sandhill Scientific Inc), which has 2 pairs of impedance electrodes in the distal esophagus, proximal esophagus, and hypopharynx (Fig. 1B). ${ }^{13}$ The top electrode in the hypopharynx was placed $0.5 \mathrm{~cm}$ proximal to the upper border of the UES under laryngoscopic guidance (Fig. 1C).

MII-pH was performed OFF PPI in patients without prior evidence of erosive esophagitis, while MII-pH was performed ON PPI when erosive esophagitis was evident prior to PPI therapy. On the other hand, all HMII were performed after at least 10-day discontinuation of PPI. The catheter was inserted through the nostril, and was connected to an ambulatory recording device (ZepHr Impedance/pH Reflux Monitoring System; Sandhill Scientific Inc). All measurements were recorded for 24 hours. Acid exposure
A

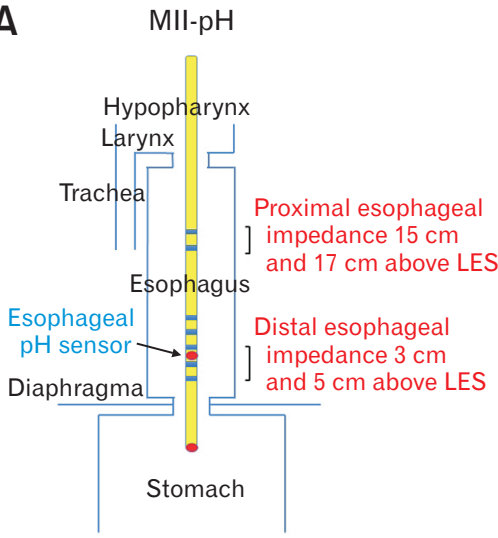

B

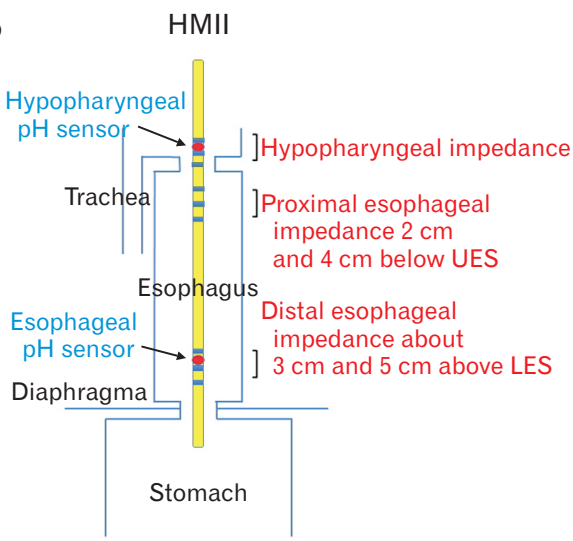

\section{Top impedance electrode}

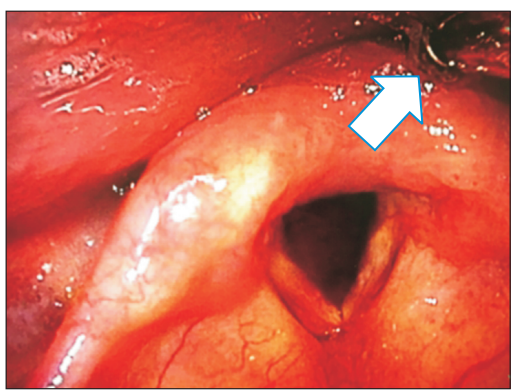

Figure 1. Location of the impedance electrode (A) A catheter for multichannel intraluminal impedance-pH (MII-pH) has impedance electrodes at $3,5,7,9,15$, and $17 \mathrm{~cm}$ and $\mathrm{pH}$ probe at $5 \mathrm{~cm}$ above the upper border of lower esophageal sphincter (LES). (B) A catheter for hypopharyngeal MII (HMII) has 3 pairs of impedance electrodes (hypopharynx, proximal esophagus; $2 \mathrm{~cm}$ and $4 \mathrm{~cm}$ below upper esophageal sphincter [UES], and distal esophagus; $21 \mathrm{~cm}$ and $23 \mathrm{~cm}$ below UES). (C) The top impedance electrode in the hypopharynx indicated by arrows was placed $0.5 \mathrm{~cm}$ proximal to the upper border of the UES.

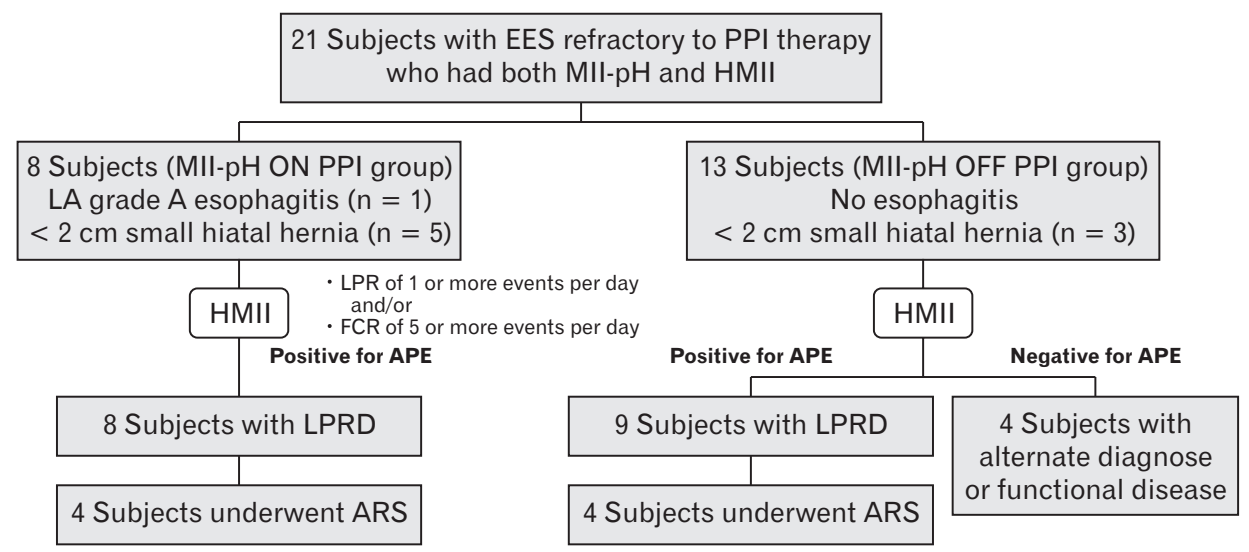

Figure 2. Flow chart of the study. EES, extraesophageal symptoms; PPI, proton pump inhibitor; MII-pH, multichannel intraluminal impedance-pH; HMII, hypopharyngeal multichannel intraluminal impedance; LA, Los Angeles; LPR, laryngopharyngeal reflux; FCR, full column reflux; APE, abnormal proximal exposure; LPRD, laryngopharyngeal reflux disease; ARS, antireflux surgery. 
time (AET) was calculated automatically, and $<4 \%$ per day was defined normal.

\section{Multichannel Intraluminal Impedance-pH and Hypopharyngeal Multichannel Intraluminal Impedance Data Interpretation}

Impedance data were analyzed by the specific software (Bioview Analysis; Sandhill Scientific Inc). The software automatically identified any possible reflux events; however each event was individually reviewed to exclude false-positive events by experienced physicians (T.M., Y.S., and T.H.). On MII-pH, a distal and a proximal reflux event was considered present when retrograde bolus transit occurred and reached the $5 \mathrm{~cm}$ and $17 \mathrm{~cm}$ impedance electrodes above the lower esophageal sphincter respectively. On HMII, an LPR event was considered present when retrograde bolus transit occurred across all ring sets and reached the hypopharynx. FCR was defined as reflux that reached the impedance $2 \mathrm{~cm}$ distal to the UES but did not reach the hypopharyngeal ring set.

\section{Symptom Association}

The association between reflux events and clinical symptoms was assessed based on the symptom index (SI) and the symptomassociation probability (SAP). The SI was considered to be positive if the value was $\geq 50 \%$. SAP values of $95 \%$ or greater were considered statistically significant. ${ }^{14}$

\section{Diagnostic Criteria Based on Multichannel Intraluminal Impedance-pH and Upper Endoscopy}

Based on the Lyon Consensus, a pathological GERD was considered present when upper endoscopy demonstrated at least 1 of the following findings: LA grade C or D esophagitis, peptic stricture or long-segment Barrett's mucosa, and/or MII-pH demonstrated AET $>6 \%$ per day. Endoscopic findings of LA grade $\mathrm{A}$ or B esophagitis and AET 4-6\% were not adequate to confirm a pathological GERD, and additional evidence such as the total number of reflux events $>80$ per day was therefore required to confirm a pathological GERD.

\section{Diagnostic Criteria Based on Hypopharyngeal Multichannel Intraluminal Impedance}

Based on the normative data established for LPR and FCR, APE was defined as LPR of 1 or more events per day and/or FCR of 5 or more events per day. ${ }^{10} \mathrm{LPRD}$ was considered present when APE was positive on HMII (Fig. 2).

\section{Antireflux Surgery and Symptom Assessment}

Laparoscopic Nissen fundoplication was a choice of ARS for the patients who had adequate esophageal motility based on highresolution manometry. To objectively assess clinical symptoms, all patients completed the RSI to quantify the nature and severity of EES the day before HMII (off PPI) and after ARS. RSI is made up of 9-items which ranges from 0 (no problem) to 5 (severe problem), with a maximum total score of 45 . Any score of greater than 13 is considered abnormal for EES. ${ }^{15}$ The outcome of ARS was assessed using before and the most recent RSI.

\section{Statistical Methods}

Values are expressed as median (range or interquartile range) except RSI score as mean \pm SD. Statistical significance was determined by Mann-Whitney test and Wilcoxon signed rank test using SPSS software program (version 22; IBM, Armonk, NY, USA). Statistical tests were 2-tailed, and a $P$-value $<0.05$ was considered statistically significant.

Table 1. Demographics for Patients With Suspected Extraesophageal Symptoms

\begin{tabular}{lc}
\hline \multicolumn{1}{c}{ Characteristics } & Subjects $(\mathrm{n}=21)$ \\
\hline Sex & \\
Male & $11 / 21(52)$ \\
Age $(\mathrm{yr})$ & $66.0[21-82]$ \\
BMI $\left(\mathrm{kg} / \mathrm{m}^{2}\right)$ & $21.6[15.8-32.7]$ \\
Primary EES & \\
Abnormal sensation in the laryngopharynx & $6 / 21(29)$ \\
Globus sensation & $5 / 21(24)$ \\
Chronic cough & $5 / 21(24)$ \\
Dysphonia & $3 / 21(14)$ \\
Halitosis & $1 / 21(5)$ \\
Sore throat & $1 / 21(5)$ \\
Esophageal mucosal injury & \\
LA grade & \\
Normal & \\
A & $20 / 21(95)$ \\
Hiatal hernia & $1 / 21(5)$ \\
RSI & $8 / 21(38)$ \\
\hline
\end{tabular}

EES, extraesophageal symptoms; BMI, body mass index; LA, Los Angeles; RSI, reflux symptom index.

Values are expressed as $\mathrm{n}(\%)$, median (range), or mean $\pm \mathrm{SD}$. 


\section{Results}

\section{Patient Demographics}

From July 1, 2015 to April 30, 2018, a total of 191 patients with EES underwent HMII. Of these 191 patients, 21 patients with EES, who had both MII-pH and HMII, were included in this study. The rest of the patients who did not undergo MII-pH prior to referral for HMII and were excluded from the further analysis. Median age and body mass index were 66.0 years (range, 21-82 years) and $21.6 \mathrm{~kg} / \mathrm{m}^{2}$ (range, $15.8-32.7 \mathrm{~kg} / \mathrm{m}^{2}$ ), respectively (Table 1). Chief complaints included abnormal sensation in the laryngopharynx $(\mathrm{n}=6 ; 29 \%)$, globus sensation $(\mathrm{n}=5 ; 24 \%)$, chronic cough ( $\mathrm{n}=5 ; 24 \%)$, dysphonia $(\mathrm{n}=3 ; 14 \%)$, halitosis $(\mathrm{n}=1 ; 5 \%)$, and sore throat $(\mathrm{n}=1 ; 5 \%)$. Twelve patients $(57 \%)$ presented with isolated EES. Nine patients (43\%) had concomitant typical GERD symptoms, and all of them had complete resolution of concomitant typical GERD symptoms with PPI therapy but continued to have EES.

\section{Upper Endoscopy and Multichannel Intraluminal Impedance-pH}

Of 21 patients, 13 patients had MII-pH OFF PPI (MII-pH OFF PPI group), while 8 patients had MII-pH ON PPI (MII$\mathrm{pH}$ ON PPI group). None of 13 patients in the MII-pH OFF PPI group had esophagitis on endoscopy and all of them had negative MII-pH. Of 8 patients in the MII-pH ON PPI group, LA

Table 2. Multichannel Intraluminal Impedance-pH and Hypopharyngeal Multichannel Intraluminal Impedance Measurements

\begin{tabular}{|c|c|c|c|c|}
\hline \multirow[b]{2}{*}{ MII-pH measurements } & \multicolumn{3}{|c|}{ Subjects } & \multirow[b]{2}{*}{$P$-value } \\
\hline & $\begin{array}{c}\text { Total } \\
(\mathrm{n}=21)\end{array}$ & $\begin{array}{c}\text { MII-pH ON PPI } \\
(\mathrm{n}=8)\end{array}$ & $\begin{array}{c}\text { MII-pH OFF PPI } \\
(\mathrm{n}=13)\end{array}$ & \\
\hline \multicolumn{5}{|l|}{ Reflux events } \\
\hline Distal reflux & $55(36-72)$ & $64.5(56-83)$ & $51(28-69)$ & 0.166 \\
\hline Proximal reflux & $23(11-39)$ & $31(18-47)$ & $15(7-29)$ & 0.057 \\
\hline AET & $0.7(0.2-1.1)$ & $0.8(0.1-1.3)$ & $0.7(0.3-1.1)$ & 0.986 \\
\hline $\mathrm{AET}>6 \%$ & $0 / 21(0)$ & $0 / 8(0)$ & $0 / 13(0)$ & \\
\hline AET $4-6 \%$ & $1 / 21(5)$ & $1 / 8(13)$ & $0 / 13(0)$ & \\
\hline $\mathrm{AET}<4 \%$ & $20 / 21(95)$ & $7 / 8(87)$ & $13 / 13(100)$ & \\
\hline \multicolumn{5}{|l|}{ Symptom index } \\
\hline Positive for EES & $1 / 21(5)$ & $1 / 8(13)$ & $0 / 13(0)$ & \\
\hline Positive for typical symptom & $4 / 21(19)$ & $1 / 8(13)$ & $3 / 13(23)$ & \\
\hline \multicolumn{5}{|l|}{ Symptom association probability } \\
\hline Positive for EES & $2 / 19(11)$ & 2/7 (29) & $0 / 12(0)$ & \\
\hline Positive for typical symptom & $2 / 19(11)$ & $1 / 7(14)$ & $1 / 12(8)$ & \\
\hline \multicolumn{5}{|l|}{ HMII measurements } \\
\hline \multicolumn{5}{|l|}{ Reflux events } \\
\hline Distal reflux & $54.5(46.5-66.5)$ & $58.5(45.5-73.5)$ & $54.0(47.0-65.0)$ & 0.276 \\
\hline FCR & $6.0(5.5-17.0)$ & $10.0(6.0-17.5)$ & $6.0(2.0-13.5)$ & 0.287 \\
\hline LPR & $0.0(0.0-1.5)$ & $0.0(0.0-2.5)$ & $0.0(0.0-1.5)$ & 0.898 \\
\hline Positive APE & $17 / 21(81)$ & $8 / 8(100)$ & $9 / 13(69)$ & 0.131 \\
\hline AET & $6.0(1.5-10.6)$ & $6.1(3.2-9.2)$ & $3.9(1.0-11.2)$ & 0.683 \\
\hline \multicolumn{5}{|l|}{ Symptom Index } \\
\hline Positive for EES & $5 / 21(24)$ & $3 / 8(38)$ & $2 / 13(15)$ & \\
\hline Positive for typical symptom & 6/21 (29) & $3 / 8(38)$ & $3 / 13(23)$ & \\
\hline \multicolumn{5}{|l|}{ Symptom association probability } \\
\hline Positive for EES & $3 / 21(14)$ & $2 / 8(25)$ & $1 / 13(8)$ & \\
\hline Positive for typical symptom & $6 / 21(29)$ & $3 / 8(38)$ & $3 / 13(23)$ & \\
\hline
\end{tabular}

MII-pH, multichannel intraluminal impedance-pH; AET, acid exposure time; EES, extraesophageal symptoms; HMII, hypopharyngeal multichannel intraluminal impedance; FCR, full column reflux; LPR, laryngopharyngeal reflux; APE, abnormal proximal exposure. 
grade A esophagitis was found in $1(5 \%)$ patient even after PPI therapy; however the number of distal reflux was $<80$ and AET was $<4 \%$. There was 1 patient without esophagitis whose AET was 4-6\%; however the number of distal reflux was $<80$. Three patients in the MII-pH OFF PPI group and 5 patients in the MII-pH ON PPI group were found to have small hiatal hernia $(<$ $2 \mathrm{~cm}$ ). No patients had peptic stricture, histology proven eosinophilic esophagitis or long-segment Barrett's esophagus. Based on this, 13 patients in the MII-pH OFF PPI group were considered to have persistent EES without presence of pathological GERD, while 8 patients in the MII-pH ON PPI group previously had a diagnosis of pathological GERD, but currently there was no more endoscopic confirmatory evidence for GERD or abnormal acid exposure to the distal esophagus due to PPI therapy. Therefore, all 21 patients were thought to have persistent EES with GERDunrelated "unknown" etiology.

\section{Laryngoscopy and Hypopharyngeal Multichannel Intraluminal Impedance}

Laryngoscopy did not show any known etiology of laryngopharyngeal symptoms in the laryngopharynx. Of 21 patients, APE was found in 17 patients (81\%) (Fig. 2); 8 patients (38\%) and 17 patients $(100 \%)$ had an abnormal number of LPR events (median, 2.5; range, 1.0-14.0) and events (median, 6.0; range, 5.0-26.0), respectively. MII-pH and HMII measurements are summarized in Table 2.

APE was identified in all 8 patients who had MII-pH ON PPI. On the other hand, APE was identified in 9 patients $(69 \%)$ out of 13 patients who had MII-pH OFF PPI. The median AET on HMII OFF PPI, MII-pH OFF PPI, and ON PPI were 6.0 (1.5-10.6), 0.7 (0.3-1.1), and 0.8 (0.1-1.3) (median [interquartile range]). Good symptom correlation with EES (both SI and SAP) was not frequently seen both in MII-pH and HMII.

\section{Antireflux Surgery}

Of 17 patients with APE, 8 patients with chronic cough $(\mathrm{n}=$ $3)$, globus sensation $(n=2)$, abnormal sensation of larynx $(n=2)$, and halitosis $(\mathrm{n}=1)$ underwent laparoscopic Nissen fundoplication. The remaining 9 patients stayed on PPI therapy. During a median follow-up of 9 months (range, 6-36 months) after ARS, 7 patients were able to discontinue PPI, although 1 patient had to resume PPI because of non-steroidal anti-inflammatory drugs use for Herpes zoster. All 8 patients had significant symptomatic improvement in the RSI scores after ARS compared to before ARS $(19.6 \pm 4.9$ pre-ARS to $5.8 \pm 1.4$ post-ARS, $P=0.008)$. RSI

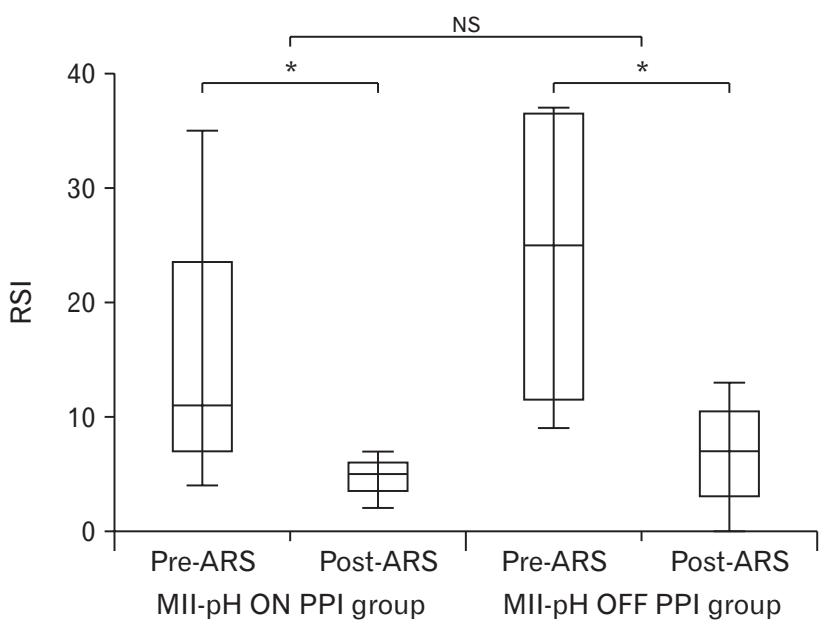

Figure 3. Comparison of the reflux symptom index (RSI) between pre- and post-antireflux surgery (ARS) in multichannel intraluminal impedance-pH (MII-pH) ON proton pump inhibitor (PPI) group and MII-pH OFF PPI group. NS, not significant. ${ }^{*} P<0.05$.

scores normalized after ARS in all patients. RSI scores changed from $15.2 \pm 13.6$ pre-ARS to $4.8 \pm 2.1$ post-ARS in patients in the MII-pH OFF PPI group $(P=0.125)$, and from $24.0 \pm 14.6$ pre-ARS to $6.8 \pm 5.4$ post-ARS in 4 patients in the MII-pH ON PPI group $(P=0.125)$. There was no difference in change values of RSI score between the groups (Fig. 3).

\section{Discussion}

This is the first study to investigate the prevalence of APE using HMII in patients with EES, who were not diagnosed with pathological GERD or were thought to have GERD-unrelated EES with "unknown" etiology based on endoscopy and MII-pH. In the present study, we demonstrated that $81 \%$ of patients with EES, who did not have objective evidence of pathological GERD or persistent GERD, had APE indicating LPRD. All patients with APE, who had undergone ARS, had significant symptomatic improvement, suggesting that endoscopy and MII-pH were not sufficient to evaluate the EES, and that the assessment of APE by HMII is essential to identify patients who could have LPRDrelated EES and would benefit from ARS.

The Lyon Consensus proposed the definitive evidence of pathological GERD such as endoscopic findings of LA grade C/ D esophagitis, esophageal stricture and long-segment Barrett's esophagus, and abnormal acid exposure to the distal esophagus as evidenced by AET $>6 \%$ based on a conventional pH-metry. Additional evidence such as the number of distal reflux events $>$ 
80 is required when upper endoscopy demonstrates LA grade A/ $\mathrm{B}$ esophagitis and AET is between $4 \%$ and $6 \% .^{3}$ As such, MII$\mathrm{pH}$ is currently a gold standard to assess acid exposure to the distal esophagus (both AET and the number of reflux events) for a diagnosis of pathological GERD, especially in patients with typical reflux symptoms such as heartburn and regurgitation. Malhotra et $\mathrm{al}^{16}$ performed MII-pH on 50 consecutive patients with EES refractory to acid suppressive therapy, and demonstrated that $16 \%$, $22 \%$, and $2 \%$ patients were found to have EES due to acid reflux, nonacid reflux or both, respectively. This suggested that the impedance technology such as MII-pH could increase a diagnostic yield by $36 \%$, compared to a conventional $\mathrm{pH}$-metry, in patients with EES. Therefore, MII-pH was also suggested to be performed in patients with GERD-related EES; however a true benefit of MII$\mathrm{pH}$ to evaluate EES has not been well discussed, and the outcome of ARS in patients with EES selected based on MII-pH remains unpredictable.

There were 2 distinct patient phenotypes in this study. Patients in the MII-pH OFF PPI group were thought not to have pathological GERD, while patients in the MII-pH ON PPI group had a prior diagnosis of pathological GERD, but there was no more endoscopic confirmatory evidence for GERD or abnormal acid exposure to the distal esophagus due to PPI therapy. The common characteristics of 2 phenotypes included (1) absence of esophageal mucosal injury, (2) normal acid exposure to the distal esophagus based on MII-pH, even with persistent EES, and (3) scarce symptom correlation between reflux events and EES on MII-pH. Based on this, this population was thought not to have NERD, which accounts for approximately $60 \%$ of GERD patients, ${ }^{17}$ or hypersensitive esophagus. Therefore, all patients were thought to have EES with GERD-unrelated "unknown" etiology. However, $81 \%$ of these patients were found to have APE on HMII indicating LPRD, and all patients with APE, who had undergone ARS, had significant symptomatic improvement. This highly suggest that LPRD may be pathophysiologically a different entity from GERD or NERD, and a conventional approach to diagnose GERD based on endoscopy and MII-pH may not be sufficient to evaluate patients with EES suggestive of LPRD.

There appears to be several unique characteristics in patients with LPRD. First, esophageal mucosal injury is not common in patients with LPRD. The previous studies have demonstrated only up to $10 \%$ of patients with LPRD had erosive esophagitis, and a majority of patients with LPRD did not have any esophageal mucosal injury. ${ }^{11,18}$ Second, patients with LPRD often have normal acid exposure to the distal esophagus. It was reported that only up to
$14 \%$ of patients with EES showed an abnormal AET on MII-pH even with laryngoscopic findings of LPRD, suggesting that both acid and non-acid reflux could cause EES. ${ }^{18,19}$ This was further supported by our previous studies, which demonstrated that a large number of APE-positive patients with EES such as chronic cough and adult-onset asthma had negative DeMeester scores and the number of total reflux events were normal. ${ }^{8,9,11}$ Third, EES is often unresponsive to PPI therapy. Several meta-analyses have demonstrated no diagnostic or therapeutic benefit of PPI to manage patients with LPRD. ${ }^{20-22}$ Lastly, a symptom correlation between reflux events and EES is often scarce. The previous study demonstrated that only 3 LPR events a week could induce severe inflammation in the hypopharynx, leading to persistent LPR symptoms, ${ }^{23}$ and this suggest that patients could be symptomatic even without actual LPR events. Because of these unique characteristics of LPRD, it is crucial to measure a direct exposure of gastric contents to the hypopharynx using HMII.

The larynx is a sensitive organ to protect lower airway from aspiration, and the network of sensory neurons is therefore abundantly distributed on laryngeal and pharyngeal epithelium. ${ }^{24}$ Any direct exposure of gastric contents to the hypopharynx regardless of whether it is acid or not, could cause EES. Since PPI therapy never reduces or stop reflux events, ARS could be theoretically an ideal treatment option for patients with EES refractory to medical therapy. ARS eliminates all types of reflux including LPR by anatomically reconstructing the antireflux barrier function at the gastroesophageal junction. We have demonstrated promising outcomes of ARS for patients with EES selected based on HMII, suggesting a potential benefit of HMII to select patients with EES for ARS. ${ }^{8,911}$ Approximately $70 \%$ of patients with EES such as chronic cough ( $\mathrm{n}=49)$ and adult-onset asthma $(\mathrm{n}=31)$ had APE, and $90 \%$ of patients with APE had symptomatic improvement after ARS., In the most recent study, we demonstrated that $73 \%$ of the patients with persistent EES despite PPI therapy $(\mathrm{n}=52)$ had APE, and all 12 patients with APE had excellent symptomatic improvement after ARS. ${ }^{11}$ Carroll et al ${ }^{25}$ used the same HMII system to select 9 patients with EES for ARS, all of whom achieved significant symptomatic improvement after ARS based on RSI (31.7 pre-ARS to 10 post-ARS, $P<0.01)$. In the present study, all 8 patients with APE had a significant symptomatic improvement after ARS, although none of them had abnormal endoscopic findings or abnormal acid exposure to the distal esophagus on MII-pH. These results suggest that a conventional diagnostic approach using endoscopy and MII-pH may not be sufficient to evaluate patients with EES, and HMII may be a reliable diagnostic tool to achieve successful treat- 
ment outcomes for LPRD.

There are some limitations to this study. First, the AET based on HMII OFF PPI was higher than that based on MII-pH. The impedance catheter for HMII was placed based on the location of hypopharyngeal impedance electrodes under laryngoscopic guidance, and the location of distal $\mathrm{pH}$ probe may not be exactly $5 \mathrm{~cm}$ proximal to the gastroesophageal junction. Therefore, the AET value may not be accurate. Depending on patients' body habitus (eg, height and length of esophagus), the AET value could be higher or lower than accurately measured. However, the criteria of APE was constructed only based on the number of hypopharyngeal exposure such as FCR and LPR, and did not include the AET value. Additionally, the recording was for only 24 hours and the results of MII-pH and HMII may vary, depending on patient's conditions. Second, we did not use some of other parameters described in the Lyon Consensus, such as gastroesophageal junction morphology, motility value of HRM test, mean nocturnal baseline impedance, and post-reflux swallow-induced peristaltic wave index in MII-pH in the present study, although all parameters need to be put together to obtain the whole picture of disease $;^{26}$ however all of these parameters could be supportive but not confirmatory for GERD. APE measured by HMII may be a promising confirmatory parameter to potentially establish the presence of LPR, causing EES. Last, the sample size was small. Further study involving a large sample size is required.

In conclusion, a conventional diagnostic approach using endoscopy and MII-pH may not be sufficient to evaluate patients with EES suggestive of LPRD, although endoscopy and MII-pH are currently the gold standard for the diagnosis of GERD. HMII is essential for diagnosing LPRD by direct measurements of abnormal proximal reflux events such as LPR that cause EES. APE as measure by HMII could be a reliable indicator for successful outcome of ARS in patients with EES.

Acknowledgements: We thank Tadanao Takeno for his technical assistance.

Financial support: This work was supported by Japan Society for the Promotion of Science Grants-in-Aid for Scientific Research (Grant No. JP18K09367).

\section{Conflicts of interest: None.}

Author contributions: Takeshi Suzuki, Yosuke Seki, Tomoaki Matsumura, Makoto Arai, Toyoyuki Hanazawa, Yoshitaka Okamoto, Haruhiko Suzuki, Kazunori Kasama, Akiko Umezawa, Yoshimoti Kurokawa, and Toshitaka Hoppo conceived and designed the experiments; Takeshi Suzuki, Yosuke Seki, and Tomoaki Matsumura performed the experiments and collected the data; Takeshi Suzuki, Yosuke Seki and Toshitaka Hoppo analyzed the data; Takeshi Suzuki and Toshitaka Hoppo wrote the manuscript; and all authors commented on previous versions of the manuscript.

\section{References}

1. Vakil N, van Zanten SV, Kahrilas P, Dent J, Jones R. The Montreal definition and classification of gastroesophageal reflux disease: a global evidence-based consensus. Am J Gastroenterol 2006;101:1900-1920.

2. Tauber S, Gross M, Issing WJ. Association of laryngopharyngeal symptoms with gastroesophageal reflux disease. Laryngoscope 2002;112:879886.

3. Gyawali CP, Kahrilas PJ, Savarino E, et al. Modern diagnosis of GERD: the Lyon consensus. Gut 2018;67:1351-1362.

4. Abou-Ismail A, Vaezi MF. Evaluation of patients with suspected laryngopharyngeal reflux: a practical approach. Curr Gastroenterol Rep 2011;13:213-218.

5. Vaezi MF. Gastroesophageal reflux-related chronic laryngitis: con. Arch Otolaryngol Head Neck Surg 2010;136:908-909.

6. Hicks DM, Ours TM, Abelson TI, Vaezi MF, Richter JE. The prevalence of hypopharynx findings associated with gastroesophageal reflux in normal volunteers. J Voice 2002;16:564-579.

7. Roman S, Gyawali CP, Savarino E, et al. Ambulatory reflux monitoring for diagnosis of gastro-esophageal reflux disease: update of the porto consensus and recommendations from an international consensus group. Neurogastroent Motil 2017;29:1-15.

8. Komatsu Y, Hoppo T, Jobe BA. Proximal reflux as a cause of adult-onset asthma: the case for hypopharyngeal impedance testing to improve the sensitivity of diagnosis. JAMA Surg 2013;148:50-58.

9. Hoppo T, Komatsu Y, Jobe BA. Antireflux surgery in patients with chronic cough and abnormal proximal exposure as measured by hypopharyngeal multichannel intraluminal impedance. JAMA Surg 2013;148:608-615.

10. Hoppo T, Sanz AF, Nason KS, et al. How much pharyngeal exposure is "normal"? normative data for laryngopharyngeal reflux events using hypopharyngeal multichannel intraluminal impedance (HMII). J Gastrointest Surg 2012;16:16-24.

11. Suzuki T, Seki Y, Okamoto Y, Hoppo T. Hypopharyngeal multichannel intraluminal impedance leads to the promising outcome of antireflux surgery in Japanese population with laryngopharyngeal reflux symptoms. Surg Endosc 2017;32:2409-2419.

12. DiBaise JK. The LA classification for esophagitis: a call for standardization. Am J Gastroenterol 1999;94:3403-3404.

13. Hoppo T, Jarido V, Pennathur A, et al. Antireflux surgery preserves lung function in patients with gastroesophageal reflux disease and endstage lung disease before and after lung transplantation. Arch Surg 2011;146:1041-1047.

14. Weusten BL, Roelofs JM, Akkermans LM, Van Berge-Henegouwen GP, Smout AJ. The symptom-association probability: an improved 
method for symptom analysis of 24-hour esophageal $\mathrm{pH}$ data. Gastroenterology 1994;107:1741-1745.

15. Belafsky PC, Postma GN, Koufman JA. Validity and reliability of the reflux symptom index (RSI). J Voice 2002;16:274-277.

16. Malhotra A, Freston JW, Aziz K. Use of $\mathrm{pH}$-impedance testing to evaluate patients with suspected extraesophageal manifestations of gastroesophageal reflux disease. J Clin Gastroenterol 2008;42:271-278.

17. Fujiwara Y, Arakawa T. Epidemiology and clinical characteristics of GERD in the Japanese population. J Gastroenterol 2009;44:518-534.

18. de Bortoli N, Nacci A, Savarino E, et al. How many cases of laryngopharyngeal reflux suspected by laryngoscopy are gastroesophageal reflux disease-related? World J Gastroenterol 2012;18:4363-4370.

19. Ang $\mathrm{D}$, Ang TL, Teo EK, et al. Is impedance $\mathrm{pH}$ monitoring superior to the conventional 24-h pH meter in the evaluation of patients with laryngorespiratory symptoms suspected to be due to gastroesophageal reflux disease? J Dig Dis 2011;12:341-348.

20. Wei C. A meta-analysis for the role of proton pump inhibitor therapy in patients with laryngopharyngeal reflux. Eur Arch Otorhinolaryngol 2016;273:3795-3801.

21. Liu C, Wang H, Liu K. Meta-analysis of the efficacy of proton pump in- hibitors for the symptoms of laryngopharyngeal reflux. Braz J Med Biol Res 2016;49:e5149.

22. Sen $P$, Georgalas C, Bhattacharyya AK. A systematic review of the role of proton pump inhibitors for symptoms of laryngopharyngeal reflux. Clin Otolaryngol 2006;31:20-24.

23. Koufman JA. The otolaryngologic manifestations of gastroesophageal reflux disease (GERD): a clinical investigation of 225 patients using ambulatory 24-hour $\mathrm{pH}$ monitoring and an experimental investigation of the role of acid and pepsin in the development of laryngeal injury. Laryngoscope 1991;101(4 pt 2 suppl 53):1-78.

24. Shiba K, Satoh I, Kobayashi N, Hayashi F. Multifunctional laryngeal motoneurons: an intracellular study in the cat. J Neurosci 1999;19:27172727.

25. Carroll TL, Nahikian K, Asban A, Wiener D. Nissen fundoplication for laryngopharyngeal reflux after patient selection using dual $\mathrm{pH}$, full column impedance testing: a pilot study. Ann Oto Rhinol Laryngol 2016;125:722-728.

26. Ghisa M, Barberio B, Savarino V, et al. The Lyon consensus: does it differ from the previous ones? J Neurogastroenterol Motil 2020;26:311321 . 